\title{
The Emerging Role of Endoscopic Ultrasound in the Diagnosis and Management of Inflammatory Bowel Disease
}

\author{
Kyle J Fortinsky ${ }^{1^{*}}$, Elaine Yong ${ }^{2}$, Brian Yan $^{3}$ and Zane R Gallinger ${ }^{1}$ \\ ${ }^{1}$ Division of Gastroenterology, University of Toronto, Toronto, Canada \\ ${ }^{2}$ Division of Gastroenterology, Sunnybrook Hospital, Toronto, Canada \\ ${ }^{3}$ Division of Gastroenterology, Western University, London, Canada
}

*Corresponding author: Kyle J Fortinsky, Division of Gastroenterology, Toronto General Hospital, Canada, E-mail: kyle.fortinsky@mail.utoronto.ca

Received date: June 30, 2016; Accepted date: July 28, 2016; Published date: August 5, 2016

Copyright: (c) 2016 Fortinsky KJ, et al. This is an open-access article distributed under the terms of the Creative Commons Attribution License, which permits unrestricted use, distribution, and reproduction in any medium, provided the original author and source are credited.

Keywords: Endoscopic ultrasound; Crohn's disease; Ulcerative colitis; Perianal disease; Pancreatico-biliary disorders

\section{Introduction}

Endoscopic ultrasound (EUS) is a rapidly evolving radiographic technique with a variety of diagnostic and therapeutic indications [1]. It has many uses, but is frequently used to assist with the management of common pancreaticobiliary disorders such as choledocholithiasis and pancreatic pseudo cysts, and to stage gastrointestinal lesions. EUS is being used to stage both intra and extra luminal cancers of the esophagus, stomach, and colon. EUS is also being used in the setting of upper gastrointestinal bleeding to target the culprit blood vessel and administer therapy [2]. EUS is also becoming increasingly useful in patients with inflammatory bowel disease (IBD) [3]. The role of EUS in patients with IBD has been explored in several small studies [4-9]. Such studies have investigated the utility of EUS as a method of distinguishing Crohn's disease (CD) from ulcerative colitis (UC) as well as assessing perianal disease. In this review, we will discuss the current evidence pertaining to EUS in IBD and possible future directions where EUS may be utilized both for diagnostic and therapeutic indications.

\section{Differentiating Crohn's Disease and Ulcerative Colitis}

The diagnosis of IBD is generally made based upon endoscopic, pathologic, and/or radiologic investigations. Most patients can be correctly categorized into CD or UC based upon this information. However, in approximately $10-15 \%$ of IBD patients, the precise diagnosis is unclear and can create uncertainty when it comes to providing certain medical and surgical treatment options [10]. Only one study to date has examined the utility of EUS in helping to clarify the diagnosis of IBD. In a prospective study of 52 patients with active IBD, Ellrichmann demonstrated the ability of EUS to differentiate between the type of IBD by identifying the relative involvement of mucosal and submucosal layers as well as adjacent lymphadenopathy [6]. Each patient underwent endoscopic biopsies in addition to a forward-viewing radial EUS of the mid-sigmoid colon. In patients with UC, the mucosal thickness was increased while the submucosa and muscularis propria were of normal thickness. Conversely, patients with CD had significant thickening only of the submucosal layer. Moreover, paracolonic lymph nodes were identified in the majority of patients with active $C D$ while lymph nodes were not identified in patients with UC (Table 1). While this study lends support to the use of EUS at distinguishing CD from UC based upon wall thickness measures of various layers, it remains unclear whether similar distinctions can be made in patients with indeterminate colitis.

\begin{tabular}{|l|l|l|l|}
\hline Type of IBD & $\begin{array}{l}\text { Mucosal } \\
\text { thickness }\end{array}$ & $\begin{array}{l}\text { Submucosal } \\
\text { thickness }\end{array}$ & $\begin{array}{l}\text { Paracolonic } \\
\text { lymphadenopathy }\end{array}$ \\
\hline $\begin{array}{l}\text { Crohn's } \\
\text { Disease }\end{array}$ & $1.32 \pm 0.17 \mathrm{~mm}$ & $2.01 \pm 0.22 \mathrm{~mm}$ & $\begin{array}{l}\text { Present in } 73 \% \text { of } \\
\text { patients }\end{array}$ \\
\hline $\begin{array}{l}\text { Ulcerative } \\
\text { colitis }\end{array}$ & $2.08 \pm 0.11 \mathrm{~mm}$ & $1.01 \pm 0.08 \mathrm{~mm}$ & $\begin{array}{l}\text { Not present in any } \\
\text { patients }\end{array}$ \\
\hline Difference & $\mathrm{P}=0.0001$ & $\mathrm{P}=0.0001$ & - \\
\hline
\end{tabular}

Table 1: Ellrichmann study comparing patients with CD and UC based upon mucosal thickening, submucosal thickening, and paracolonic lymphadenopathy as seen on mid-sigmoid colon using forwardviewing radial EUS.

\section{Assessing Disease Activity}

EUS may be used to assess disease activity in IBD, thereby guiding treatment decisions and monitoring response to treatment. Ellrichmann demonstrated significantly greater wall thickness in patients with active CD or UC $(3.51 \mathrm{~mm}, 95 \%$ confidence interval [CI] 2.36-2.66 mm) compared to patients with $\mathrm{CD}$ or UC who were in remission (1.76 mm, 95\% CI 1.74-1.78 mm). Notably, total wall thickness and histological inflammation on biopsies were strongly correlated ( $r=0.43$ for UC, $r=0.69$ for $\mathrm{CD}$ ) [6]. In another prospective study, Tsuga performed EUS in the rectum of 72 patients with documented UC and compared them to 36 healthy controls. Tsuga confirmed that total wall thickness was highly predictive for acute inflammation in patients with UC compared to healthy controls and that the degree of rectal wall involvement correlated with endoscopic severity [11]. A scoring system was developed to better characterize disease activity in the rectum in patients with UC (Table 2). This scoring system allows for more objective descriptions of the rectum and promotes more objective definitions of disease activity (Figures 1 and 2).

In a prospective study of 23 patients with quiescent UC as defined by clinical symptoms and endoscopic findings, Higaki performed a baseline rectal EUS to determine wall thickness [12]. All patients were maintained on medical therapy (either sulfasalazine $2 \mathrm{~g} /$ day or 5 aminosalicylic acid $1.5 \mathrm{~g} /$ day and were followed for 1 year to monitor for signs of clinical or endoscopic relapse. Higaki et al. identified 8 patients who relapsed during this time, while the remainder of patients were in remission. Interestingly, the patients who relapsed during the first year had significantly greater rectal wall thickening of the first 3 layers seen on their baseline EUS $(2.73 \mathrm{~mm}, 95 \%$ CI $2.13-3.33 \mathrm{~mm})$ compared to those patients who remained in remission $(1.79 \mathrm{~mm}, 95 \%$ 
CI 1.56-1.99 mm; $\mathrm{P}=0.0001$ ). This study provides evidence that EUS may be able to identify patients with UC who are more likely to relapse and therefore warrant more aggressive treatment and/or monitoring.

\begin{tabular}{|l|l|l|l|}
\hline Score & Wall thickening & $\begin{array}{l}\text { Mucosa-submucosa } \\
\text { border }\end{array}$ & $\begin{array}{l}\text { Submucosa- } \\
\text { muscularis propria } \\
\text { border }\end{array}$ \\
\hline I & Absent & Smooth & Smooth \\
\hline II & Present & Smooth & Smooth \\
\hline IIIa & Present & Irregular & Smooth \\
\hline IIIb & Present & Irregular & Irregular \\
\hline IVa & Present & Blurred & Smooth \\
\hline IVb & Present & Blurred & Irregular \\
\hline
\end{tabular}

Table 2: Scoring system developed by Tsuga et al. to describe ultrasound findings in patients with active UC.

The diagnosis of IBD is generally made based upon endoscopic, pathologic, and/or radiologic investigations. Most patients can be correctly categorized into CD or UC based upon this information. However, in approximately $10-15 \%$ of IBD patients, the precise diagnosis is unclear and can create uncertainty when it comes to providing certain medical and surgical treatment options [10]. Only one study to date has examined the utility of EUS in helping to clarify the diagnosis of IBD. In a prospective study of 52 patients with active IBD, Ellrichmann demonstrated the ability of EUS to differentiate between the type of IBD by identifying the relative involvement of mucosal and submucosal layers as well as adjacent lymphadenopathy [6]. Each patient underwent endoscopic biopsies in addition to a forward-viewing radial EUS of the mid-sigmoid colon. In patients with UC, the mucosal thickness was increased while the submucosa and muscularis propria were of normal thickness. Conversely, patients with $\mathrm{CD}$ had significant thickening only of the submucosal layer. Moreover, paracolonic lymph nodes were identified in the majority of patients with active $C D$ while lymph nodes were not identified in patients with UC (Table 1). While this study lends support to the use of EUS at distinguishing CD from UC based upon wall thickness measures of various layers, it remains unclear whether similar distinctions can be made in patients with UC who are more likely to relapse and therefore warrant more aggressive treatment and/or monitoring.

In an elegant study performed both in vitro and in vivo, Yoshizawa further delineated the role of EUS in identifying the depth of inflammation in patients with UC [13]. In vitro, they demonstrated that the degree of vertical spread of intestinal inflammation as seen on EUS correlated to histopathological findings in $90 \%$ of cases studied (45 of 50 specimens). More specifically, the concordance rate was $95 \%$ for inflammation in the submucosa, $83 \%$ for inflammation extending to the muscularis propria, and $100 \%$ for inflammation extending to the subserosa. Moreover, the concordance rate was higher for sites with open ulcers $(100 \%, 21 / 21)$ compared to sites with ulcer scars $(83 \%$, 24/29; $\mathrm{P}=0.045$ ). Yoshizawa explained that the imperfect concordance was related to the imperfect sensitivity of EUS to identify localized inflammation deep in the intestinal wall. As part of the in vivo study, Yoshizawa studied 42 patients with UC and demonstrated that the depth of inflammation as seen on EUS was correlated with clinical severity. In 27 patients whereby EUS identified inflammation only to the submucosa, clinical severity was deemed mild to moderate in 24 patients (89\%) and severe in 3 patients (11\%). Conversely, in 15 patients whereby EUS identified inflammation extending to the muscularis propria or deeper, clinical severity was deemed severe in 7 patients (47\%). Importantly, of these 15 patients with more extensive inflammation, $10(67 \%)$ required colectomy while only $5(19 \%)$ responded to induction therapy. EUS may therefore play a role in predicting clinical outcomes and guiding disease management.th indeterminate colitis.

In the largest observational study to date involving 200 patients with UC, Hurlstone correlated EUS scores with histopathology as well as endoscopic and clinical scores [14]. Hurlstone demonstrated that findings on EUS taken throughout the colon did correlate with endoscopic, clinical, and histopathologic scoring systems (kappa values ranging from 0.48-0.78). Lastly, a large prospective study of 67 patients with UC and 46 patients with CD evaluated the utility of contrastenhanced trans abdominal ultrasound in evaluating disease activity [15]. Overall, Socaciu found that contrast-enhanced abdominal ultrasound correlated slightly better with endoscopic disease activity than clinical scoring systems alone and therefore represents a potentially non-invasive way to monitor disease in these patients. Unfortunately, there are no studies to compare EUS-guided images and trans abdominally obtained images to determine which is more effective at guiding treatment. Furthermore, one limitation for most of the aforementioned studies is the lack of blinding to clinical and endoscopic information while performing the EUS, which may have biased some of the results.

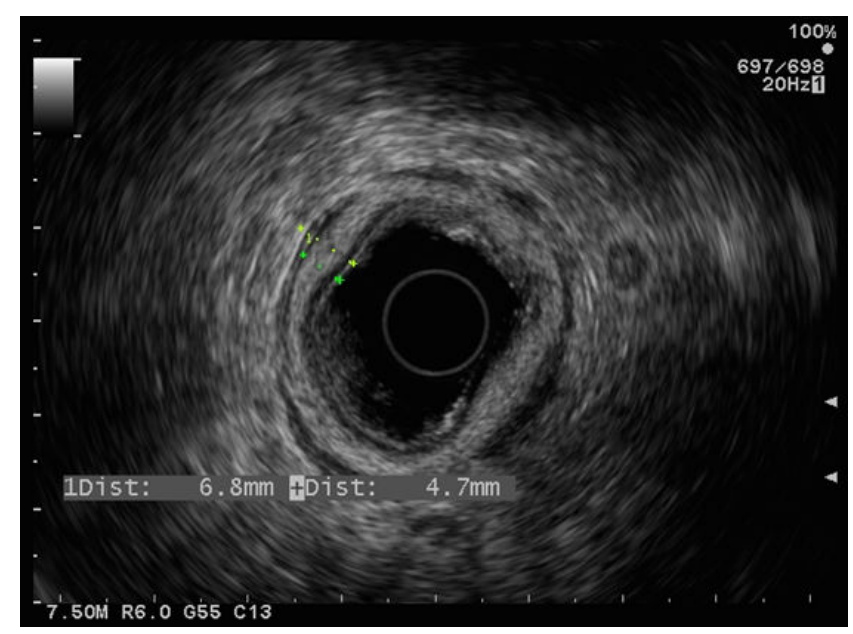

Figure 1: Radial EUS of rectum in a patient with UC demonstrating increased total wall thickness with smooth borders between mucosa and submucosa, and between submucosa and muscularis propria corresponding to a Tsuga Score of 2. 


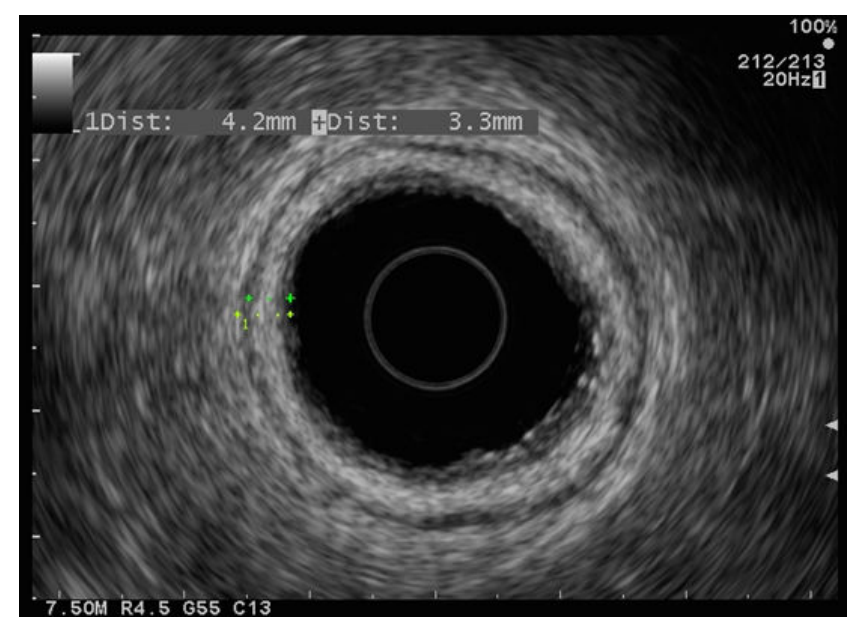

Figure 2: Radial EUS of rectum in a patient with UC demonstrating increased total wall thickness, blurred border between mucosa and submucosa, and irregular border between submucosa and muscularis propria corresponding to a Tsuga Score of $4 \mathrm{~b}$.

\section{Perianal Disease}

Recent guidelines for the diagnosis of fistulizing perianal Crohn's disease recommend the use of high resolution MRI or EUS for disease assessment [16]. While data for the use of MRI and EUA (exam under anesthesia) in the diagnosis of perianal disease more robust, small studies have shown excellent diagnostic accuracy of EUS in perianal disease, as well as its potential role in guiding medical management. A prospective study by Schwartz randomized thirty-four patients with CD for evaluation of perianal disease with EUS, MRI or EUA. EUS and EUA demonstrated the highest accuracy for detecting fistula anatomy at $91 \%$ (91\%, CI $75 \%-98 \%)$, followed by MRI, which was $87 \%$ accurate (CI 69\%-96\%) When pelvic MRI or rectal EUS was combined with surgical evaluation, accuracy increased to $100 \%$ [9]. Another prospective study by Spradlin attempted to use EUS to help guide therapy for the treatment of perianal CD. Patients randomized to the EUS group had their medical and surgical management guided by results of EUS at 22 and 38 weeks, with cessation of drainage as the primary outcome. Patients in the control group had EUS when enrolled, as well as at completion of the study. Decisions regarding care of the control group was at the discretion of the surgeon. [8]. While the sample size of the study was small (ten patients), the intervention group showed a trend towards earlier closure of fistulas. Wiese randomized twenty-four patients with perianal Crohn's disease to serial EUS with standard versus standard care alone. Medical treatment was escalated in the EUS group if the fistulas did not demonstrate improvement or had increased in size. Patients in the EUS groups demonstrated higher rate of drainage cessation at 24 weeks, but this was lost at 48 weeks. While still not routinely used, it is foreseeable that EUS will be added to the armamentarium of imaging tools available to the gastroenterologist in the management of perianal disease.

\section{Characterization of Dysplastic Lesions}

Another role for EUS is the evaluation of potentially dysplastic mucosal lesions. Current guidelines suggest that patients with chronic
UC undergo dysplasia surveillance, which should be performed using either chromoendosocopy or multiple random biopsies [17]. Patients with high-grade dysplasia are often referred for colectomy, and if there is an endoscopically visible lesion, an attempt at endoscopic mucosal resection may be considered. Decisions surrounding the management of low-grade dysplasia can be more challenging. The most recent guidelines released by the European Crohn's and Colitis Organization suggest a multidisciplinary, detailed team discussion to determine if the patient should proceed with a colectomy or repeat chromoendosocopy with additional random biopsies within 3 months [18]. While it is not recommended in guidelines, EUS may be able to accurately detect depth of invasion, and subsequently better inform these decisions. A prospective study of 13 patients demonstrated good correlation between EUS estimation of the deepest layer of invasion compared with histopathologic depth [5]. After determining the depth of invasion using EUS, the tumours were removed using EMR or colectomy. EUS was able to accurately predict the depth of invasion in 15 of the 16 tumours. This study suggests EUS might be able to assist with the often difficult decision of whether or not to attempt removal of the dysplastic lesion with EMR, or send for a definitive colectomy. Nonetheless, the utility of this approach is not well understood and should be used cautiously.

\section{Future Directions}

As the field of diagnostic and therapeutic EUS continues to evolve, its utility in patients with IBD will grow. EUS may soon provide an alternative modality to abdominal ultrasound, CT, or MRI for identifying evidence of active inflammation. Moreover, EUS may be used to augment current endoscopic scoring systems and even reduce the need for biopsies in certain circumstances. Another possible use of EUS could be in the classification and management of Crohn's related strictures. Specifically, EUS may be able to distinguish malignant from benign strictures and to identify whether a lesion represents acute or chronic inflammation. EUS may also help to provide more accurate localization of abnormal tissue that may warrant a biopsy. Lastly, as therapeutic EUS techniques continue to progress, it is possible that gastroenterologists may be able to manage perianal fistulas and abscesses by placing Setons under endoscopic ultrasound guidance without the need for surgical consultation and examination under anaesthesia. More collaboration and research is needed between gastroenterologists with an interest in IBD and therapeutic endoscopists to further define the role of both diagnostic and therapeutic EUS in the management of patients with IBD.

\section{References}

1. Shergill AK, Lightdale JR, Bruining DH, Acosta RD, Chandrasekhara V, et al. (2015) The role of endoscopy in inflammatory bowel disease. Gastrointest Endosc 81: 1101-1121.

2. Barthet M (2012) Use of endoscopic ultrasound for management of severe, refractory gastrointestinal bleeding. Gastroenterol Hepatol 8: 678-680.

3. Lew RJ, Ginsberg GG (2002) The role of endoscopic ultrasound in inflammatory bowel disease. Gastrointestinal endoscopy clinics of North America 12: 561-571.

4. Wiese DM, Beaulieu D, Slaughter JC, Horst S, Wagnon J, et al. (2015) Use of Endoscopic Ultrasound to Guide Adalimumab Treatment in Perianal Crohn's Disease Results in Faster Fistula Healing. Inflamm Bowel Dis 21: 1594-1599. 
Citation: Fortinsky KJ, Yong E, Yan B, Gallinger ZR (2016) The Emerging Role of Endoscopic Ultrasound in the Diagnosis and Management of Inflammatory Bowel Disease. J Inflam Bowel Dis \& Disord 1: 109.

Page 4 of 4

5. Kobayashi K, Kawagishi K, Ooka S, Yokoyama K, Sada M, et al. (2015) Clinical usefulness of endoscopic ultrasonography for the evaluation of ulcerative colitis-associated tumors. WJG 21: 2693-2699.

6. Ellrichmann M, Wietzke-Braun P, Dhar S, Nikolaus S, Arlt A, et al. (2014) Endoscopic ultrasound of the colon for the differentiation of Crohn's disease and ulcerative colitis in comparison with healthy controls. Alimentary pharmacology \& therapeutics 39: 823-833.

7. Rustemovic N, Cukovic-Cavka S, Brinar M, Radic D, Opacic M, et al. (2011) A pilot study of transrectal endoscopic ultrasound elastography in inflammatory bowel disease. BMC Gastroenterol 11: 113.

8. Spradlin NM, Wise PE, Herline AJ, Muldoon RL, Rosen M, et al. (2008) A randomized prospective trial of endoscopic ultrasound to guide combination medical and surgical treatment for Crohn's perianal fistulas. The American journal of gastroenterology 103: 2527-2535.

9. Schwartz DA, Wiersema MJ, Dudiak KM, Fletcher JG, Clain JE, et al. (2001) A comparison of endoscopic ultrasound, magnetic resonance imaging, and exam under anesthesia for evaluation of Crohn's perianal fistulas. Gastroenterology 121: 1064-1072.

10. Odze RD (2015) A contemporary and critical appraisal of 'indeterminate colitis'. Mod Pathol 28: S30-S46.

11. Tsuga K, Haruma K, Fujimura J, Hata J, Tani H, et al. (1998) Evaluation of the colorectal wall in normal subjects and patients with ulcerative colitis using an ultrasonic catheter probe. Gastrointest Endosc 48: 477-484.

12. Higaki S, Nohara H, Saitoh Y, Akazawa A, Yanai H, et al. (2002) Increased rectal wall thickness may predict relapse in ulcerative colitis: a pilot follow-up study by ultrasonographic colonoscopy. Endoscopy 34: 212-219.

13. Yoshizawa S, Kobayashi K, Katsumata T, Saigenji K, Okayasu I (2007) Clinical usefulness of EUS for active ulcerative colitis. Gastrointest Endosc 65: 253-260.

14. Hurlstone DP, Sanders DS, Lobo AJ, McAlindon ME, Cross SS (2005) Prospective evaluation of high-frequency mini-probe ultrasound colonoscopic imaging in ulcerative colitis: a valid tool for predicting clinical severity. Eur J Gastroenterol Hepatol 17: 1325-1331.

15. Socaciu M, Ciobanu L, Diaconu B, Hagiu C, Seicean A, et al. (2015) NonInvasive Assessment of Inflammation and Treatment Response in Patients with Crohn's Disease and Ulcerative Colitis using ContrastEnhanced Ultrasonography Quantification. J Gastrointestin Liver Dis 24: 457-465.

16. Ong EM, Ghazi LJ, Schwartz DA, Mortele KJ (2015) Crohn's Colitis Foundation of America I. Guidelines for imaging of Crohn's perianal fistulizing disease. Inflamm Bowel Dis 21: 731-736.

17. Laine L, Kaltenbach T, Barkun A, McQuaid KR, Subramanian V, et al. (2015) SCENIC international consensus statement on surveillance and management of dysplasia in inflammatory bowel disease. Gastrointestinal endoscopy 81: 489-501.

18. Annese V, Daperno M, Rutter MD, Amiot A, Bossuyt P, et al. (2013) European evidence based consensus for endoscopy in inflammatory bowel disease. J Crohns Colitis 7: 982-1018. 